\title{
Microdroplet Mass Spectrometry Enables Extremely Accelerated Pepsin Digestion of Proteins
}

\author{
Tobias Rainer, ReinerEidelpes, Martin Tollinger and Thomas Müller* \\ Institute of Organic Chemistry and Centerfor Molecular Biosciences (CMBI), Leopold- \\ Franzens University Innsbruck, 6020 Innsbruck, Austria \\ *E-mail: thomas.mueller@uibk.ac.at
}

\section{Supporting Information}

\section{Data processing}

The obtained full MS raw data (acquisition time $1 \mathrm{~min}$ ) were averaged, exported as .raw files using Xcalibur Qual Browser 4.1 software (Thermo Scientific, San Jose, CA), and converted to mzML format using the open source software MSconvert V3.0. ${ }^{1}$ The open source tool mMass V5.5.0 $0^{2}$ was used for peak picking, in silico protein digestion and peptide identification. The mMass sequence function was used to calculate all possible peptide signals up to charge state $8+$ (allowing variable methionine oxidation). The computed peptides were matched with the spectra and annotated (mass tolerance $\pm 5 \mathrm{ppm}$ ). All fitted signals were checked manually for isotope, charge and $\mathrm{m} / \mathrm{z}$ accuracy. Non-matching annotations we re deleted.

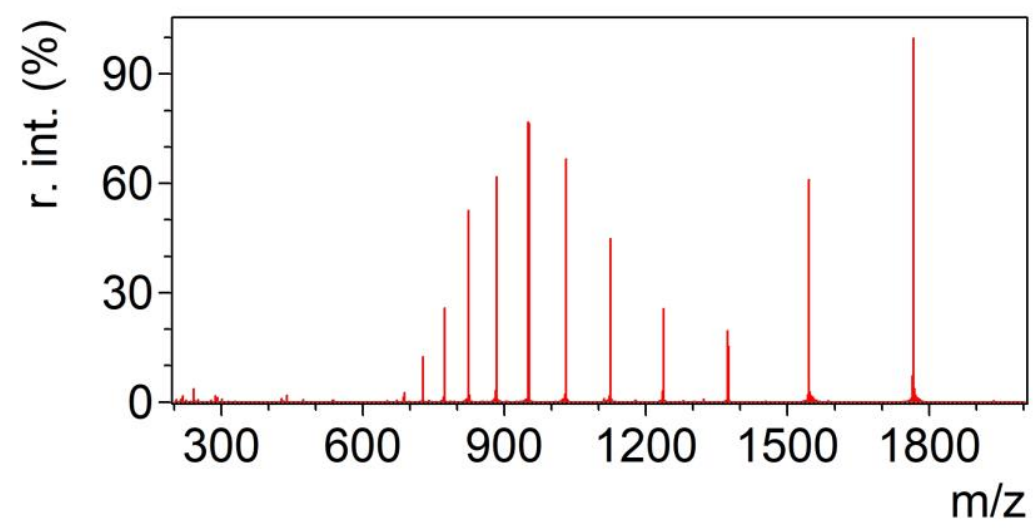

Figure S1. ESSI-Spectrum of cytochrome c sample Cyt3 mixed 1:1 (v/v) with 20\% acetic acid. 


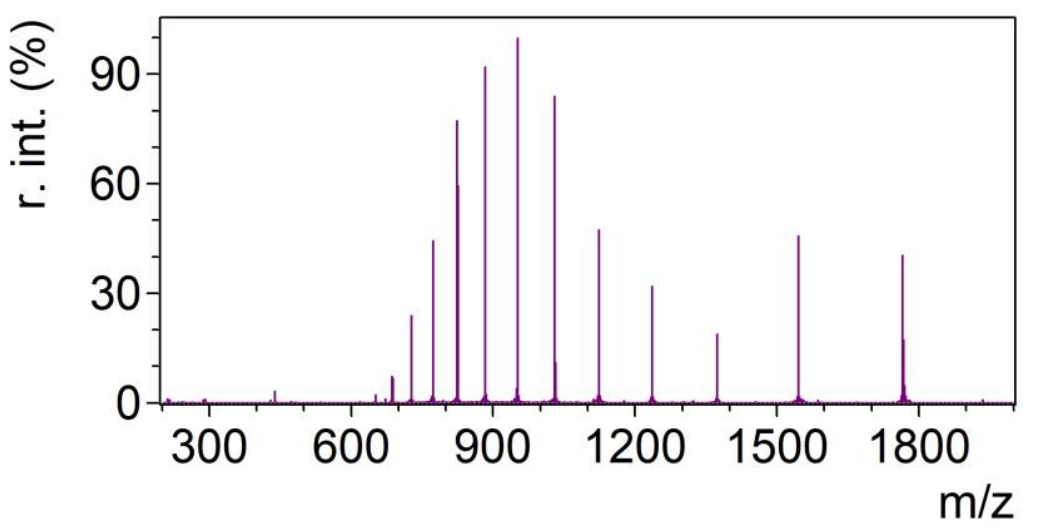

Figure S2. ESSI-Spectrum of cytochrome c sample Cyt3 mixed 1:1 (v/v) with 8\% formic acid.

\section{Annotation of peptides obtained by in-spray digestion of cytochrome c}

In-spray digestion of cytochrome $\mathrm{c}$ using ESSI-MS3: experimental setup as depicted in Figures $1 \mathrm{~A}, \mathrm{~S} 6$ and S7, DoE Run N16 showed the best results in terms of sequence coverage at the following settings: flow rate $5 \mu \mathrm{L} \cdot \mathrm{min}^{-1}, 7 \mathrm{~cm}$ distance between sprayer and MS inlet, sample Cyt3, protease solution Pep3, 120 psi nitrogen back pressure. The obtained sequence coverage was $98.1 \%$, matched intensity was found to be $24.1 \%$.

Cytochrome c from horse heart: Sequence length 105, average mass $11.880 \mathrm{kDa}$, single letter code sequence: MGDVEKGKKI FVQKCAQCHT VEKGGKHKTG PNLHGLFGRK TGQAPGFTYT DANKNKGITW KEETLMEYLE NPKKYIPGTK MIFAGIKKKT EREDLIAYLK KATNE

Table S1. Annotated peptides found for in-spray digestion of cytochrome c in DoE run N16. Mass tolerance was set to $\pm 5 \mathrm{ppm}$.

\begin{tabular}{|l|l|l|}
\hline $\mathbf{m} / \mathbf{z}$ meas. & $\mathbf{z}$ & \multicolumn{1}{|c|}{ Annotation } \\
\hline 402.2345 & 2 & {$[3-9]$ g.DVEKGKK.i } \\
\hline 463.2682 & 3 & {$[69-80]$ y.LENPKKYIPGTK.m } \\
\hline 463.2682 & 3 & {$[84-95]$ f.AGI KKKTEREDL.i } \\
\hline 483.7668 & 2 & {$[98-105]$ a.YLKKATNE. } \\
\hline 509.7753 & 2 & {$[38-47]$ f.GRKTGQAPGF.t } \\
\hline 509.7753 & 2 & {$[37-46]$ I.FGRKTGQAPG.f } \\
\hline 524.6426 & 3 & {$[84-97]$ f.AGI KKKTEREDLIA.y } \\
\hline 549.9862 & 3 & {$[82-95]$ m.IFAGIKKKTEREDL.i } \\
\hline 549.9862 & 3 & {$[83-96]$ i.FAGIKKKTEREDLI.a } \\
\hline 561.3044 & 3 & {$[68-81]$ e.YLENPKKYIPGTKM.i } \\
\hline 575.8275 & 2 & {$[96-105]$ I.IAYLKKATNE. } \\
\hline 602.2832 & 2 & {$[14-24]$ q.KCAQCHTVEKG.g } \\
\hline 611.3598 & 3 & {$[82-97]$ m.IFAGIKKKTEREDLIA.y } \\
\hline 630.6102 & 4 & {$[84-105]$ f.AGIKKKTEREDLIAYLKKATNE. } \\
\hline 648.0197 & 3 & {$[68-83]$ e.YLENPKKYIPGTKMIF.a } \\
\hline 662.9703 & 5 & {$[68-95]$ e.YLENPKKYIPGTKMIFAGIKKKTEREDL.i } \\
\hline 694.3991 & 2 & {$[69-80]$ y.LENPKKYIPGTK.m } \\
\hline 694.3991 & 2 & {$[84-95]$ f.AGI KKKTEREDL.i } \\
\hline 695.6486 & 4 & {$[82-105]$ m.IFAGIKKKTEREDLIAYLKKATNE. } \\
\hline
\end{tabular}




\begin{tabular}{|c|c|c|}
\hline 699.7959 & 5 & [70-99] I.ENPKKYIPGTKMIFAGIKKKTEREDLIAYL.K \\
\hline 699.7959 & 5 & [69-98] y.LENPKKYIPGTKMIFAGIKKKTEREDLIAY.I \\
\hline 699.7959 & 5 & [68-97] e.YLENPKKYIPGTKMIFAGIKKKTEREDLIA.y \\
\hline 714.9864 & 5 & [65-94] t.LMEYLENPKKYIPGTKMIFAGIKKKTERED.I \\
\hline 714.9864 & 5 & [66-95] I.MEYLENPKKYIPGTKMIFAGIKKKTEREDL.i \\
\hline 734.7158 & 3 & [66-83] I.MEYLENPKKYIPGTKMIF.a \\
\hline 741.2498 & 6 & [67-104] m.EYLENPKKYIPGTKMIFAGIKKKTEREDLIAYLKKATN.e \\
\hline 741.2498 & 6 & [68-105] e.YLENPKKYIPGTKMIFAGIKKKTEREDLIAYLKKATNE. \\
\hline 751.8097 & 5 & [66-97] I.MEYLENPKKYIPGTKMIFAGIKKKTEREDLIA.y \\
\hline 778.65 & 4 & [38-65] f.GRKTGQAPGFTYTDANKNKGITWKEETL.m \\
\hline 784.5966 & 6 & [66-105] I.MEYLENPKKYIPGTKMIFAGIKKKTEREDLIAYLKKATNE. \\
\hline 786.4593 & 2 & [84-97] f.AGIKKKKTEREDLIA.y \\
\hline 824.4746 & 2 & [83-96] i.FAGIKKKTEREDLI.a \\
\hline 828.4612 & 4 & [68-95] e.YLENPKKYIPGTKMIFAGIKKKTEREDL.i \\
\hline 843.6737 & 4 & [38-67] f.GRKTGQAPGFTYTDANKNKGITWKEETLME.y \\
\hline 860.7349 & 4 & [68-96] e.YLENPKKYIPGTKMIFAGIKKKTEREDLI.a [1xOxidation] \\
\hline 874.4914 & 4 & [68-97] e.YLENPKKYIPGTKMIFAGIKKKTEREDLIA.y \\
\hline 874.4914 & 4 & [69-98] y.LENPKKYIPGTKMIFAGIKKKTEREDLIAY.I \\
\hline 874.4914 & 4 & [70-99]I.ENPKKYIPGTKMIFAGIKKKTEREDLIAYL.K \\
\hline 889.297 & 5 & [67-104] m.EYLENPKKYIPGTKMIFAGIKKKTEREDLIAYLKKATN.e \\
\hline 889.297 & 5 & [68-105] e.YLENPKKYIPGTKMIFAGIKKKTEREDLIAYLKKATNE. \\
\hline 893.4803 & 4 & [65-94] t.LMEYLENPKKYIPGTKMIFAGIKKKTERED.I \\
\hline 893.4803 & 4 & [66-95] I.MEYLENPKKYIPGTKMIFAGIKKKTEREDL.i \\
\hline 927.197 & 3 & [82-105] m.IFAGIKKKTEREDLIAYLKKATNE. \\
\hline 939.5118 & 4 & [66-97] I.MEYLENPKKYIPGTKMIFAGIKKKTEREDLIA.y \\
\hline 941.3125 & 5 & [66-105] I.MEYLENPKKYIPGTKMIFAGIKKKTEREDLIAYLKKATNE. \\
\hline 966.5257 & 1 & [98-105] a.YLKKATNE. \\
\hline 971.5269 & 2 & [68-83] e.YLENPKKYIPGTKMIF.a \\
\hline 980.5207 & 4 & [3-38] g.DVEKGKKIFVQKCAQCHTVEKGGKHKTGPNLHGLFG.r \\
\hline 1037.87 & 3 & [38-65] f.GRKTGQAPGFTYTDANKNKGITWKEETL.m \\
\hline 1101.5704 & 2 & [66-83] I.MEYLENPKKYIPGTKMIF.a \\
\hline 1104.2797 & 3 & [68-95] e.YLENPKKYIPGTKMIFAGIKKKTEREDL.i \\
\hline 1111.3697 & 4 & [67-104] m.EYLENPKKYIPGTKMIFAGIKKKTEREDLIAYLKKATN.e \\
\hline 1111.3697 & 4 & [68-105] e.YLENPKKYIPGTKMIFAGIKKKTEREDLIAYLKKATNE. \\
\hline 1143.5936 & 3 & [35-65] h.GLFGRKTGQAPGFTYTDANKNKGITWKEETL.m \\
\hline 1150.6476 & 1 & [96-105] I.IAYLKKATNE. \\
\hline 1165.6556 & 3 & [69-98] y.LENPKKYIPGTKMIFAGIKKKTEREDLIAY.I \\
\hline 1165.6556 & 3 & [68-97] e.YLENPKKYIPGTKMIFAGIKKKTEREDLIA.y \\
\hline 1165.6556 & 3 & [70-99]I.ENPKKYIPGTKMIFAGIKKKTEREDLIAYL.K \\
\hline 1176.3945 & 4 & [66-105] I.MEYLENPKKYIPGTKMIFAGIKKKTEREDLIAYLKKATNE. \\
\hline 1252.3497 & 3 & [66-97] I.MEYLENPKKYIPGTKMIFAGIKKKTEREDLIA.Y \\
\hline 1481.4962 & 3 & [67-104] m.EYLENPKKYIPGTKMIFAGIKKKTEREDLIAYLKKATN.e \\
\hline 1481.4962 & 3 & [68-105] e.YLENPKKYI PGTKMIFAGIKKKTEREDLIAYLKKATNE. \\
\hline 1568.1904 & 3 & [66-105] I.MEYLENPKKYIPGTKMIFAGIKKKTEREDLIAYLKKATNE. \\
\hline
\end{tabular}




\section{Annotation of peptides obtained by in-spray digestion of recombinant $\mathrm{RocC}_{24-126}$}

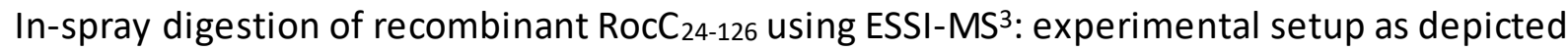
in Figures 1A, S6 and S7, flow rate was $1 \mu \mathrm{L} \cdot \mathrm{min}^{-1}, 5 \mathrm{~cm}$ distance between sprayer and MS inlet, sample RocC1 (approx. $0.26 \mathrm{mg} \cdot \mathrm{mL}^{-1}$ in $5 \mathrm{mM} \mathrm{NH}_{4} \mathrm{HCO}_{3}$ ), protease solution Pep3, $70 \mathrm{psi}$ nitrogen back pressure. Obtained sequence coverage was $100 \%$, matched intensity was found to be $60.3 \%$.

RocC $_{24-126}$ (in-house, recombinant with protease tag GPLGS): Sequence length 108, 12.069 kDa average mass, single letter code: GPLGSARSDA LLWLAANFPE AFDNSLRIRP LKIGIMSDIL QHAEKAEQVG VSKSKLREAV VLFTRRLDYL ACLKAREVRI DLHGNPVAEV TEEEAENASM KIKKRVEK

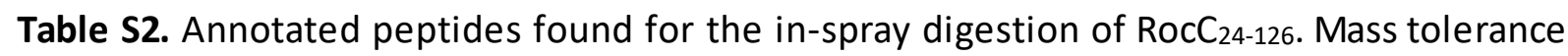
was set to \pm 5 ppm.

\begin{tabular}{|c|c|c|}
\hline $\mathrm{m} / \mathrm{z}$ meas. & $\mathbf{z}$ & Annotation \\
\hline 399.5923 & 3 & [27-36] I.RIRPLKIGIM.s \\
\hline 431.2644 & 1 & [11-13] a.LLW.I \\
\hline 431.2644 & 1 & [12-14] I.LWL.a \\
\hline 437.287 & 3 & [26-36] s.LRIRPLKIGIM.s \\
\hline 464.5047 & 4 & [63-77] I.FTRRLDYLACLKARE.v \\
\hline 466.9457 & 3 & [27-38]I.RIRPLKIGIMSD.i \\
\hline 468.7663 & 2 & [64-70] f.TRRLDYL.a \\
\hline 504.6402 & 3 & [27-39] I.RIRPLKIGIMSDI.I \\
\hline 504.6402 & 3 & [26-38] s.LRIRPLKIGIMSD.i \\
\hline 522.277 & 2 & [1-11] .GPLGSARSDAL.I \\
\hline 542.3001 & 2 & [62-69] v.LFTRRLDY.I \\
\hline 542.3001 & 2 & [63-70] I.FTRRLDYL.a \\
\hline 555.5472 & 4 & [90-108] e.VTEEEAENASMKIKKRVEK. \\
\hline 563.3188 & 4 & [39-58] d.ILQHAEKAEQVGVSKSKLRE.a \\
\hline 574.0513 & 7 & [26-61] s.LRIRPLKIGIMSDILQHAEKAEQVGVSKSKLREAVV.I \\
\hline 574.0513 & 7 & [27-62] I.RIRPLKIGIMSDILQHAEKAEQVGVSKSKLREAVVL.f \\
\hline 578.8189 & 2 & [1-12] .GPLGSARSDALL.w \\
\hline 585.5032 & 5 & [83-108] I.HGNPVAEVTEEEAENASMKIKKRVEK. \\
\hline 587.4816 & 6 & [78-108] e.VRIDLHGNPVAEVTEEEAENASMKIKKRVEK. \\
\hline 598.8853 & 2 & [27-36] I.RIRPLKIGIM.s \\
\hline 601.9979 & 3 & [74-89] I.KAREVRIDLHGNPVAE.v \\
\hline 612.331 & 2 & [64-73] f.TRRLDYLACL.K \\
\hline 615.3817 & 1 & [78-82] e.VRIDL.h \\
\hline 619.0037 & 3 & [63-77] I.FTRRLDYLACLKARE.v \\
\hline 629.3229 & 2 & [63-72] I.FTRRLDYLAC.I \\
\hline 631.9421 & 5 & [63-89] I.FTRRLDYLACLKAREVRIDLHGNPVAE.v \\
\hline 639.692 & 3 & [73-89] c.LKAREVRIDLHGNPVAE.v \\
\hline 658.8831 & 4 & [39-62] d.ILQHAEKAEQVGVSKSKLREAVVL.f \\
\hline 660.3564 & 2 & [78-89] e.VRIDLHGNPVAE.v \\
\hline 660.3564 & 2 & [79-90] v.RIDLHGNPVAEV.t \\
\hline 660.3564 & 2 & [77-88] r.EVRIDLHGNPVA.e \\
\hline 668.1937 & 6 & [74-108] I.KAREVRIDLHGNPVAEVTEEEAENASMKIKKRVEK. \\
\hline 669.5581 & 6 & [26-61] s.LRIRPLKIGIMSDILQHAEKAEQVGVSKSKLREAVV.I \\
\hline 669.5581 & 6 & [27-62]I.RIRPLKIGIMSDILQHAEKAEQVGVSKSKLREAVVL.f \\
\hline 670.4848 & 8 & $\begin{array}{l}\text { [62-107] v.LFTRRLDYLACLKAREVRIDLHGNPVAEVTEEEAENASMKIKKRVE.K } \\
\text { [1xOxidation] }\end{array}$ \\
\hline 685.8648 & 2 & [62-72] v.LFTRRLDYLAC.I \\
\hline 685.8648 & 2 & [63-73] I.FTRRLDYLACL.k \\
\hline 695.6495 & 4 & [39-63] d.ILQHAEKAEQVGVSKSKLREAVVLF.t \\
\hline
\end{tabular}




\begin{tabular}{|c|c|c|}
\hline 697.7075 & 3 & [71-89] I.ACLKAREVRIDLHGNPVAE.v \\
\hline 699.9144 & 2 & [27-38] I.RIRPLKIGIMSD.i \\
\hline 704.7758 & 5 & [78-108] e.VRIDLHGNPVAEVTEEEAENASMKIKKRVEK. \\
\hline 704.7758 & 5 & [75-105] k.AREVRIDLHGNPVAEVTEEEAENASMKIKKR.v \\
\hline 709.3965 & 4 & [37-62] m.SDILQHAEKAEQVGVSKSKLREAVVL.f \\
\hline 709.3965 & 4 & [29-54] i.RPLKIGIMSDILQHAEKAEQVGVSKS.k \\
\hline 716.0476 & 6 & [71-108] I.ACLKAREVRIDLHGNPVAEVTEEEAENASMKI KKRVEK. \\
\hline 728.3989 & 2 & [1-14] .GPLGSARSDALLWL.a \\
\hline 731.6265 & 4 & [83-108] I.HGNPVAEVTEEEAENASMKIKKRVEK. \\
\hline 740.3927 & 3 & [90-108] e.VTEEEAENASMKIKKRVEK. \\
\hline 746.1635 & 4 & [37-63] m.SDILQHAEKAEQVGVSKSKLREAVVLF.t \\
\hline 754.7302 & 3 & [74-93] I.KAREVRIDLHGNPVAEVTEE.e \\
\hline 765.9813 & 7 & $\begin{array}{l}\text { [63-108] I.FTRRLDYLACLKAREVRIDLHGNPVAEVTEEEAENASMKIKKRVEK. } \\
\text {. }\end{array}$ \\
\hline 789.6762 & 4 & $\begin{array}{l}\text { [63-89] I.FTRRLDYLACLKAREVRIDLHGNPVAE.V } \\
\end{array}$ \\
\hline 799.1556 & 7 & [12-61] I.LWLAANFPEAFDNSLRIRPLKIGIMSDILQHAEKAEQVGVSKSKLREAVV.I \\
\hline 799.1556 & 7 & [13-62] I.WLAANFPEAFDNSLRIRPLKIGIMSDILQHAEKAEQVGVSKSKLREAVVL.f \\
\hline 801.6303 & 5 & [74-108] I.KAREVRIDLHGNPVAEVTEEEAENASMKIKKRVEK. \\
\hline 803.2675 & 5 & [27-62] I.RIRPLKIGIMSDILQHAEKAEQVGVSKSKLREAVVL.f \\
\hline 803.2675 & 5 & [26-61] s.LRIRPLKIGIMSDILQHAEKAEQVGVSKSKLREAV.I \\
\hline 815.3086 & 7 & [12-62] I.LWLAANFPEAFDNSLRIRPLKIGIMSDILQHAEKAEQVGVSKSKLREAVVL.f \\
\hline 815.3086 & 7 & [11-61] a.LLWLAANFPEAFDNSLRIRPLKIGIMSDILQHAEKAEQVGVSKSKLLREAVV.I \\
\hline 824.2471 & 5 & [73-108] c.LKAREVRIDLHGNPVAEVTEEEAENASMKIKKRVEK. \\
\hline 825.8841 & 5 & [26-62] s.LRIRPLKIGIMSDILQHAEKAEQVGVSKSKLREAVLL.f \\
\hline 859.0562 & 5 & [71-108] I.ACLKAREVRIDLHGNPVAEVTEEEEAENASMKI KKRVEK. \\
\hline 878.174 & 3 & [39-62] d.ILQHAEKAEQVGVSKSKLLREAVVL.f \\
\hline 880.7174 & 4 & [78-108] e.VRIDLHGNPVAEVTEEEAENASMKIKKRVEK. \\
\hline 880.7174 & 4 & [75-105] k.AREVRIDLHGNPVAEVTEEEAENASMKIKKR.v \\
\hline 882.3185 & 6 & [14-61] w.LAANFPEAFDNSLRIRPLKIGIMSDILQHAEKAEQVGVSKSKLREAVV.I \\
\hline 882.3185 & 6 & [15-62] I.AANFPEAFDNSLRIRPLKIGIMSDILQHAEKAEQVGVSKSKLREAVVL.f \\
\hline 892.4681 & 3 & [15-38] I.AANFPEAFDNSLRIRPLKIGIMSD.i \\
\hline 902.4922 & 2 & [74-89] I.KAREVRIDLHGNPVAE.v \\
\hline 908.5202 & 3 & [49-72] q.VGVSKSKLREAVVLFTRRLDYLAC.I \\
\hline 916.5159 & 3 & [38-62] s.DI LQHAEKAEQVGVSKSKLREAVVL.f \\
\hline 916.5159 & 3 & [29-53] i.RPLKIGIMSDILQHAEKAEQVGVSK.s \\
\hline 918.5185 & 5 & [22-62] a.FDNSLRIRPLKIGIMSDILQHAEKAEQVGVSKSKLREAVVL.f \\
\hline 918.5185 & 5 & [23-63] f.DNSLRIRPLKIGIMSDILQHAEKAEQVGVSKSKLREAVVLF.t \\
\hline 924.8355 & 3 & [13-36] I.WLAANFPEAFDNSLRIRPLKIGIM.S \\
\hline 927.1973 & 3 & [39-63] d.ILQHAEKAEQVGVSKSKLREAVVLF.t \\
\hline 928.0018 & 2 & [63-77] I.FTRRLDYLACLKARE.v \\
\hline 932.1803 & 6 & [12-61] I.LWLAANFPEAFDNSLRIRPLKIGIMSDILQHAEKAEQVGVSKSKLREAVV.I \\
\hline 932.1803 & 6 & [13-62] I.WLAANFPEAFDNSLRIRPLKIGIMSDILQHAEKAEQVGVSKSKLREAVVL.f \\
\hline 945.5266 & 3 & [37-62] m.SDILQHAEKAEQVGVSKSKLREAVVL.f \\
\hline 945.5266 & 3 & [29-54] i.RPLKIGIMSDILQHAEKAEQVGVSKS.k \\
\hline 951.0258 & 6 & [11-61] a.LLWLAANFPEAFDNSLRIRPLKIGIMSDILQHAEKAEQVGVSKSKLREAVV.I \\
\hline 951.0258 & 6 & [12-62] I.LWLAANFPEAFDNSLRIRPLKIGIMSDILQHAEKAEQVGVSKSKLREAVVL.f \\
\hline 953.8486 & 3 & [13-37] I.WLAANFPEAFDNSLRIRPLKIGIMS.d \\
\hline 962.5306 & 3 & [12-36] I.LWLAANFPEAFDNSLRIRPLKIGIM.S \\
\hline 988.0431 & 2 & [22-38] a.FDNSLRIRPLKIGIMSD.i \\
\hline 992.1894 & 3 & [13-38] I.WLAANFPEAFDNSLRIRPLKIGIMSD.i \\
\hline 994.55 & 3 & [37-63] m.SDILQHAEKAEQVGVSKSKLREAVVLF.t \\
\hline 1001.7867 & 4 & 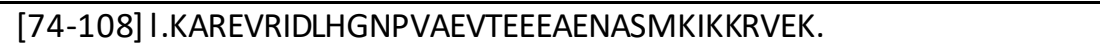 \\
\hline 1003.8326 & 4 & [26-61] s.LRIRPLKIGIMSDILQHAEKAEQVGVSKSKLREAVV.I \\
\hline 1003.8326 & 4 & [27-62] I.RIRPLKIGIMSDILQHAEKAEQVGVSKSKLREAVVL.f \\
\hline 1018.4966 & 1 & [13-21]I.WLAANFPEA.f \\
\hline 1029.8843 & 3 & [12-38] I.LWLAANFPEAFDNSLRIRPLKIGIMSD.i \\
\hline 1032.1044 & 4 & $\begin{array}{l}\text { [26-62] s.LRIRPLKIGIMSDILQHAEKAEQVGVSKSKLREAVVL.f } \\
\end{array}$ \\
\hline
\end{tabular}




\begin{tabular}{|c|c|c|}
\hline 1041.9611 & 5 & [13-58] I.WLAANFPEAFDNSLRIRPLKIGIMSDILQHAEKAEQVGVSKSKLRE.a \\
\hline 1043.5452 & 1 & [1-11] .GPLGSARSDAL.I \\
\hline 1052.5654 & 3 & [63-89] I.FTRRLDYLACLKAREVRIDLHGNPVAE.v \\
\hline 1058.5824 & 5 & [15-62] I.AANFPEAFDNSLRIRPLKIGIMSDILQHAEKAEQVGVSKSKLREAVVL.f \\
\hline 1058.5824 & 5 & [14-61] w.LAANFPEAFDNSLRIRPLKIGIMSDILQHAEKAEQVGVSKSKLREAVV.I \\
\hline 1064.5872 & 5 & $\begin{array}{l}\text { [11-57] a.LLWLAANFPEAFDNSLRIRPLKIGIMSDILQHAEKAEQVGVSKSKLR.e } \\
\text { [1xOxidation] }\end{array}$ \\
\hline 1064.5872 & 5 & $\begin{array}{l}\text { [12-58] I.LWLAANFPEAFDNSLRIRPLKIGIMSDILQHAEKAEQVGVSKSKLRE.a } \\
\end{array}$ \\
\hline 1071.9703 & 5 & $\begin{array}{l}\text { [63-108] I.FTRRLDYLACLKAREVRIDLHGNPVAEVTEEEAENASMKIKKRVEK. } \\
\text {. }\end{array}$ \\
\hline 1073.5692 & 4 & [71-108] I.ACLKAREVRIDLHGNPVAEVTEEEAENASMKI KKRVEK. \\
\hline 1083.5921 & 1 & [62-69] v.LFTRRLDY.I \\
\hline 1083.5921 & 1 & [63-70] I.FTRRLDYL.a \\
\hline 1118.4151 & 5 & $\begin{array}{l}\text { [12-61] I.LWLAANFPEAFDNSLRIRPLKIGIMSDILQHAEKAEQVGVSKSKLREAVV.I } \\
\end{array}$ \\
\hline 1118.4151 & 5 & [13-62]I.WLAANFPEAFDNSLRIRPLKIGIMSDILQHAEKAEQVGVSKSKLREAVVL.f \\
\hline 1131.5825 & 1 & [12-21] I.LWLAANFPEA.f \\
\hline 1141.0311 & 5 & [11-61] a.LLWLAANFPEAFDNSLRIRPLKIGIMSDILQHAEKAEQVGVSKSKLREAVV.I \\
\hline 1141.0311 & 5 & [12-62] I.LWLAANFPEAFDNSLRIRPLKIGIMSDILQHAEKAEQVGVSKSKLREAVVL.f \\
\hline 1156.6291 & 1 & [1-12] .GPLGSARSDALL.w \\
\hline 1165.5665 & 1 & [13-22] I.WLAANFPEAF.d \\
\hline 1173.9557 & 3 & $\begin{array}{l}\text { [78-108] e.VRIDLHGNPVAEVTEEEAENASMKIKKRVEK. } \\
\text {. }\end{array}$ \\
\hline 1188.1325 & 2 & [73-93] c.LKAREVRIDLHGNPVAEVTEE.e \\
\hline 1205.6342 & 4 & [1-44] .GPLGSARSDALLWLAANFPEAFDNSLRIRPLKIGIMSDILQHAE.k [1xOxidation] \\
\hline 1223.655 & 1 & [64-73] f.TRRLDYLACL.K \\
\hline 1257.6382 & 1 & [63-72] I.FTRRLDYLAC.I \\
\hline 1295.5874 & 1 & [14-25] w.LAANFPEAFDNS.I \\
\hline 1295.5874 & 1 & [15-26] I.AANFPEAFDNSL.r \\
\hline 1316.7594 & 2 & [39-62] d.I LQHAEKAEQVGVSKSKLREAVVL.f \\
\hline 1319.7061 & 1 & [77-88] r.EVRIDLHGNPVA.e \\
\hline 1319.7061 & 1 & [78-89] e.VRIDLHGNPVAE.V \\
\hline 1319.7061 & 1 & [79-90] v.RIDLHGNPVAEV.t \\
\hline 1322.979 & 4 & [14-61] w.LAANFPEAFDNSLRIRPLKIGIMSDILQHAEKAEQVGVSKSKLREAVV.I \\
\hline 1322.979 & 4 & [15-62] I.AANFPEAFDNSLRIRPLKIGIMSDILQHAEKAEQVGVSKSKLREAVVL.f \\
\hline 1335.3819 & 3 & [74-108] I.KAREVRIDLHGNPVAEVTEEEAENASMKIKKRVEK. \\
\hline 1338.112 & 3 & [26-61] s.LRIRPLKIGIMSDILQHAEKAEQVGVSKSKLREAVV.I \\
\hline 1338.112 & 3 & [27-62] I.RIRPLKIGIMSDILQHAEKAEQVGVSKSKLREAVVL.f \\
\hline 1339.7417 & 4 & $\begin{array}{l}\text { [33-79] k.IGIMSDILQHAEKAEQVGVSKSKLREAVVLFTRRLDYLACLKAREVR.i } \\
\text { [1xOxidation] }\end{array}$ \\
\hline 1370.7241 & 1 & [62-72] v.LFTRRLDYLAC.I \\
\hline 1370.7241 & 1 & [63-73] I.FTRRLDYLACL.k \\
\hline 1373.0769 & 3 & [36-71] i.MSDILQHAEKAEQVGVSKSKLREAVVLFTRRLDYLA.c [1xOxidation] \\
\hline 1373.0769 & 3 & [73-108] c.LKAREVRIDLHGNPVAEVTEEEAENASMKIKKRVEK. \\
\hline 1386.7541 & 2 & [13-36] I.WLAANFPEAFDNSLRIRPLKIGIM.s \\
\hline 1390.2917 & 2 & [39-63] d.ILQHAEKAEQVGVSKSKLREAVVLF.t \\
\hline 1397.7701 & 4 & [12-61] I.LWLAANFPEAFDNSLRIRPLKIGIMSDILQHAEKAEQVGVSKSKLREAVV.I \\
\hline 1397.7701 & 4 & [13-62] I.WLAANFPEAFDNSLRIRPLKIGIMSDILQHAEKAEQVGVSKSKLREAVVL.f \\
\hline 1417.7884 & 2 & [37-62] m.SDILQHAEKAEQVGVSKSKLREAVVL.f \\
\hline 1417.7884 & 2 & [29-54] i.RPLKIGIMSDILQHAEKAEQVGVSKS.k \\
\hline 1426.0414 & 4 & [11-61] a.LLWLAANFPEAFDNSLRIRPLKIGIMSDILQHAEKAEQVGVSKSKLREAVV.I \\
\hline 1426.0414 & 4 & [12-62]I.LWLAANFPEAFDNSLRIRPLKIGIMSDILQHAEKAEQVGVSKSKLREAVVL.f \\
\hline 1431.0911 & 3 & [71-108] I.ACLKAREVRIDLHGNPVAEVTEEEAENASMKI KKRVEK. \\
\hline 1443.297 & 2 & [12-36] I.LWLAANFPEAFDNSLRIRPLKIGIM.s \\
\hline 1455.7971 & 1 & [1-14] .GPLGSARSDALLWL.a \\
\hline 1481.669 & 1 & [13-25] I.WLAANFPEAFDNS.I \\
\hline 1487.7849 & 2 & [13-38] I.WLAANFPEAFDNSLRIRPLKIGIMSD.i \\
\hline 1544.3245 & 2 & [12-38] I.LWLAANFPEAFDNSLRIRPLKIGIMSD.i \\
\hline 1544.3245 & 2 & [13-39] I.WLAANFPEAFDNSLRIRPLKIGIMSDI.I \\
\hline
\end{tabular}




\begin{tabular}{|c|l|l|}
\hline 1594.754 & 1 & {$[12-25]$ I.LWLAANFPEAFDNS.I } \\
\hline 1594.754 & 1 & {$[13-26]$ I.WLAANFPEAFDNSL.r } \\
\hline 1707.8404 & 1 & {$[11-25]$ a.LLWLAANFPEAFDNS.I } \\
\hline 1707.8404 & 1 & {$[12-26]$ I.LWLAANFPEAFDNSL.r } \\
\hline
\end{tabular}

\section{Design of Experiment (DoE) screening}

For the DoE screening model, the input parameters were set as flow rate $\left(1,2.5,5 \mu \mathrm{l} \cdot \mathrm{min}^{-1}\right)$, distance $(3,5,7 \mathrm{~cm})$, sample solution (Cyt1, Cyt2, Cyt3), protease solution (Pep1, Pep2, Pep3), N2 pressure $(60,90,120 \mathrm{psi})$. Output parameters were defined: sequence coverage, matched intensity, number of identified peptides, average peptide length. A half fractional factorial interaction model with 3 center points was used, giving a $5+$ resolution design with 19 runs in total.

Table S3. Experimental parameters and results of DoE runs.

\begin{tabular}{|c|c|c|c|c|c|c|c|c|c|c|}
\hline $\begin{array}{l}\text { Exp } \\
\text { Name }\end{array}$ & $\begin{array}{l}\text { Run } \\
\text { Order }\end{array}$ & $\begin{array}{l}\text { Flow rate } \\
/ \mu \mid \cdot \min ^{-1}\end{array}$ & $\begin{array}{l}\text { Distance } \\
\text { /cm }\end{array}$ & $\begin{array}{l}\text { Sample } \\
\text { solution }\end{array}$ & $\begin{array}{l}\text { Protease } \\
\text { solution }\end{array}$ & $\begin{array}{c}\text { Gas } \\
\text { pressure } \\
\text { /psi }\end{array}$ & $\begin{array}{c}\text { Sequence } \\
\text { coverage } \\
/ \%\end{array}$ & $\begin{array}{c}\text { Matched } \\
\text { intensity } \\
\text { /\% }\end{array}$ & $\begin{array}{c}\text { Number } \\
\text { of } \\
\text { peptides } \\
\text { detected }\end{array}$ & $\begin{array}{c}\text { Average } \\
\text { peptide } \\
\text { length }\end{array}$ \\
\hline N1 & 3 & 1 & 3 & Cyt1 & Pep1 & 120 & 39 & 11.2 & 33 & 29 \\
\hline N2 & 15 & 5 & 3 & Cyt1 & Pep1 & 60 & 39 & 1.2 & 9 & 36 \\
\hline N3 & 11 & 1 & 7 & Cyt1 & Pep1 & 60 & 39 & 10.8 & 34 & 29 \\
\hline N4 & 1 & 5 & 7 & Cyt1 & Pep1 & 120 & 39 & 7.2 & 24 & 29 \\
\hline N5 & 8 & 1 & 3 & Cyt3 & Pep1 & 60 & 39 & 6.6 & 32 & 28 \\
\hline N6 & 10 & 5 & 3 & Cyt3 & Pep1 & 120 & 39 & 4 & 17 & 32 \\
\hline N7 & 2 & 1 & 7 & Cyt3 & Pep1 & 120 & 39 & 12 & 32 & 30 \\
\hline N8 & 19 & 5 & 7 & Cyt3 & Pep1 & 60 & 39 & 3.2 & 15 & 33 \\
\hline N9 & 16 & 1 & 3 & Cyt1 & Pep3 & 60 & 51.4 & 4.7 & 27 & 33 \\
\hline N10 & 14 & 5 & 3 & Cyt1 & Pep3 & 120 & 39 & 8.8 & 27 & 29 \\
\hline N11 & 18 & 1 & 7 & Cyt1 & Pep3 & 120 & 39 & 23.7 & 40 & 29 \\
\hline N12 & 17 & 5 & 7 & Cyt1 & Pep3 & 60 & 39 & 8.7 & 28 & 31 \\
\hline N13 & 12 & 1 & 3 & Cyt3 & Pep3 & 120 & 45.7 & 17.5 & 53 & 27 \\
\hline N14 & 13 & 5 & 3 & Cyt3 & Pep3 & 60 & 40 & 5.3 & 35 & 30 \\
\hline N15 & 4 & 1 & 7 & Cyt3 & Pep3 & 60 & 96.2 & 13.2 & 50 & 27 \\
\hline N16 & 5 & 5 & 7 & Cyt3 & Pep3 & 120 & 98.1 & 24.7 & 65 & 25 \\
\hline N17 & 9 & 2.5 & 5 & Cyt2 & Pep2 & 90 & 39 & 11.9 & 25 & 31 \\
\hline N18 & 6 & 2.5 & 5 & Cyt2 & Pep2 & 90 & 42.9 & 11.1 & 25 & 28 \\
\hline N19 & 7 & 2.5 & 5 & Cyt2 & Pep2 & 90 & 43.8 & 7.9 & 21 & 28 \\
\hline
\end{tabular}




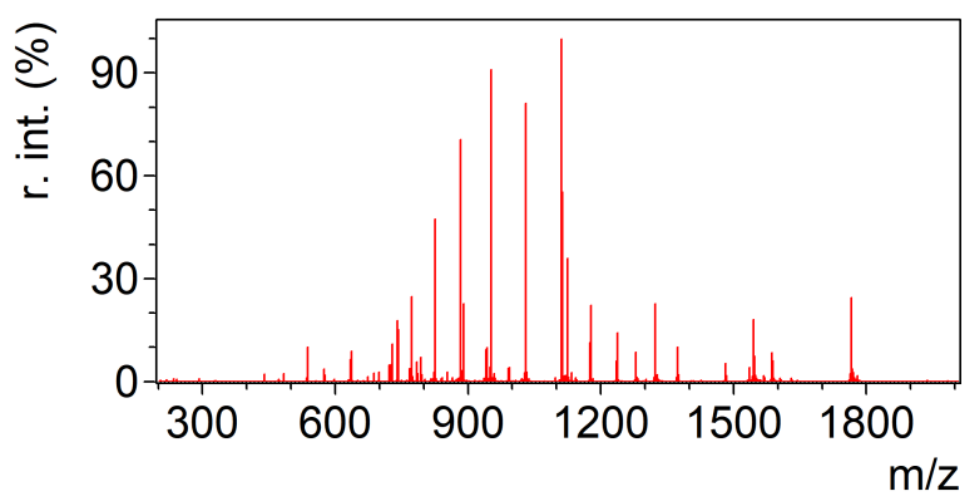

Figure S3. ESSI-MS spectrum of the worst performing DoE run (N2) of in-spray digestion of cytochrome c. Experimental setup as depicted in Figure $1 \mathrm{~A}$, flow rate was $5 \mu \mathrm{L} \cdot \mathrm{min}^{-1}, 3 \mathrm{~cm}$ distance between sprayer and MS inlet, sample Cyt1, protease solution Pep1, 60 psi nitrogen back pressure.

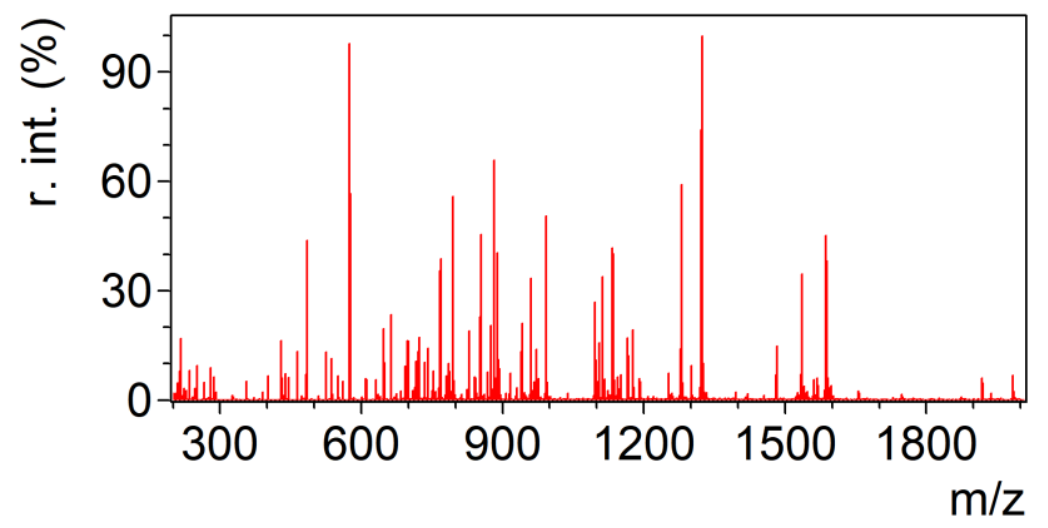

Figure S4. ESSI-MS spectrum of the best performing DoE run (N16) of in-spray digestion of cytochrome c. Experimental setup as depicted in Figure $1 \mathrm{~A}$ flow rate was $5 \mu \mathrm{L} \cdot \mathrm{min}^{-1}, 7 \mathrm{~cm}$ distance between sprayer and MS inlet, sample Cyt3, protease solution Pep3, 120 psi nitrogen back pressure. 


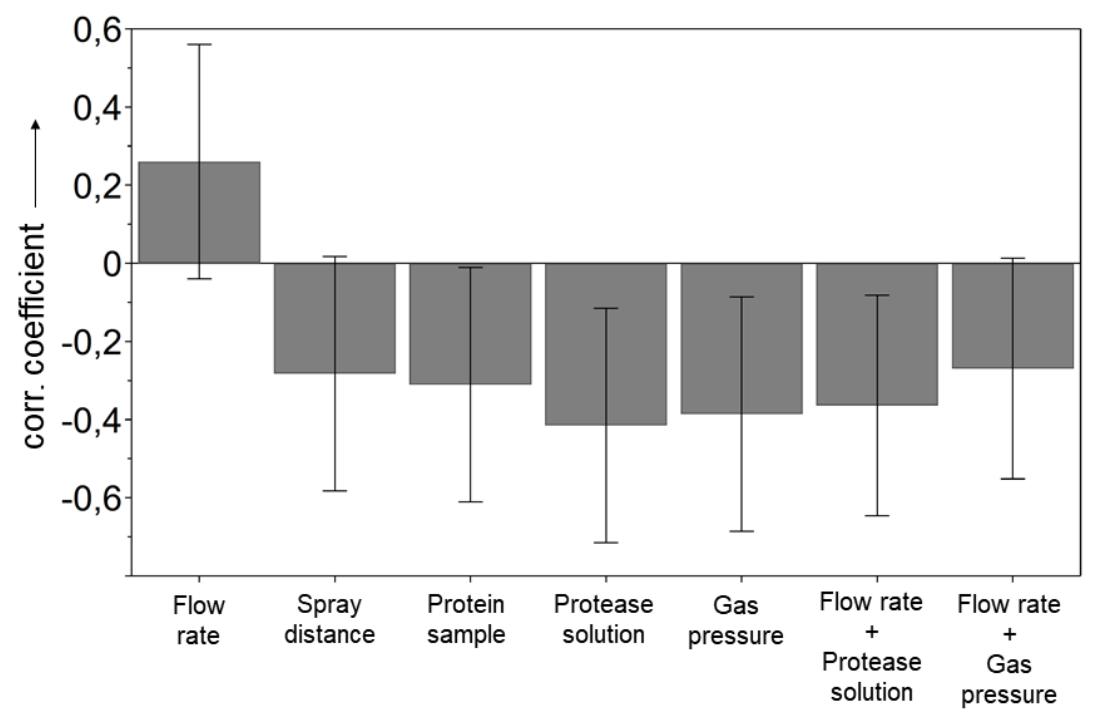

Figure S5. Result of DoE screening. Normalized coefficients plot for the output variable average peptide length obtained by an analysis of 19 randomized runs ( $R 2=0.80, N=19$, DF $=11$, confidence: $95 \%$ ). For further details see the Experimental Section and Tables S3 and S5.

Table S4. DoE analysis wizard (MODDE ${ }^{\circledR}$ Pro 12) information for the output variable sequence coverage.

\begin{tabular}{|c|c|c|c|c|}
\hline Sequence coverage & Coeff.SC & Std. Err. & $\mathbf{P}$ & Conf. int( \pm ) \\
\hline Constant & 46.6368 & 0.997194 & $4.67767 e-12$ & 2.25583 \\
\hline Flow rate & -0.954595 & 1.02452 & 0.375785 & 2.31764 \\
\hline Distance & 5.66864 & 1.02452 & 0.000364334 & 2.31764 \\
\hline Sample solution & 6.57609 & 1.02452 & 0.00012255 & 2.31764 \\
\hline Protease solution & 8.03745 & 1.02452 & $2.58686 \mathrm{e}-05$ & 2.31764 \\
\hline Gas pressure & -0.282843 & 1.02452 & 0.788726 & 2.31764 \\
\hline Flow rate*Gas pressure & 6.72222 & 0.965926 & $6.61559 e-05$ & 2.1851 \\
\hline Distance*Sample solution & 6.72222 & 0.965926 & $6.6156 \mathrm{e}-05$ & 2.1851 \\
\hline Distance*Protease solution & 5.34445 & 0.965926 & 0.000364334 & 2.1851 \\
\hline Sample*Protease solution & 6.2 & 0.965926 & 0.000122551 & 2.1851 \\
\hline $\mathrm{N}=19$ & $\mathrm{Q} 2=$ & 0.691 & Cond. no. = & 1.061 \\
\hline $\mathrm{DF}=9$ & $\mathrm{R} 2=$ & 0.971 & RSD = & 4.347 \\
\hline Comp. $=1$ & $\mathrm{R} 2$ adj. = & 0.942 & Confidence $=$ & 0.95 \\
\hline
\end{tabular}


Table S5. DoE analysis wizard (MODDE ${ }^{\circledR}$ Pro 12 ) information for the output variable average peptide length.

\begin{tabular}{|c|c|c|c|c|}
\hline Average peptide length & Coeff. SC & Std. Err. & $\mathbf{P}$ & Conf. int( \pm ) \\
\hline Constant & 29.6037 & 0.342325 & $6.16342 \mathrm{e}-17$ & 0.753442 \\
\hline Flow rate & 0.671751 & 0.351705 & 0.0825427 & 0.774088 \\
\hline Distance & -0.729498 & 0.351705 & 0.062322 & 0.774088 \\
\hline Sample solution & -0.802567 & 0.351705 & 0.0433888 & 0.774088 \\
\hline Protease solution & -1.07245 & 0.351705 & 0.0110618 & 0.774088 \\
\hline Gas pressure & -0.997021 & 0.351705 & 0.0162316 & 0.774088 \\
\hline Flow rate*Protease solution & -0.94 & 0.331591 & 0.0162316 & 0.729817 \\
\hline Flow rate*Gas pressure & -0.695556 & 0.331591 & 0.0598451 & 0.729818 \\
\hline$N=19$ & $\mathrm{Q} 2=$ & 0.547 & Cond.no. $=$ & 1.061 \\
\hline $\mathrm{DF}=11$ & $\mathrm{R} 2=$ & 0.796 & $\mathrm{RSD}=$ & 1.492 \\
\hline Comp. $=1$ & $\mathrm{R} 2 \mathrm{adj} .=$ & 0.666 & Confidence $=$ & 0.95 \\
\hline
\end{tabular}

\section{Experimental Setup}

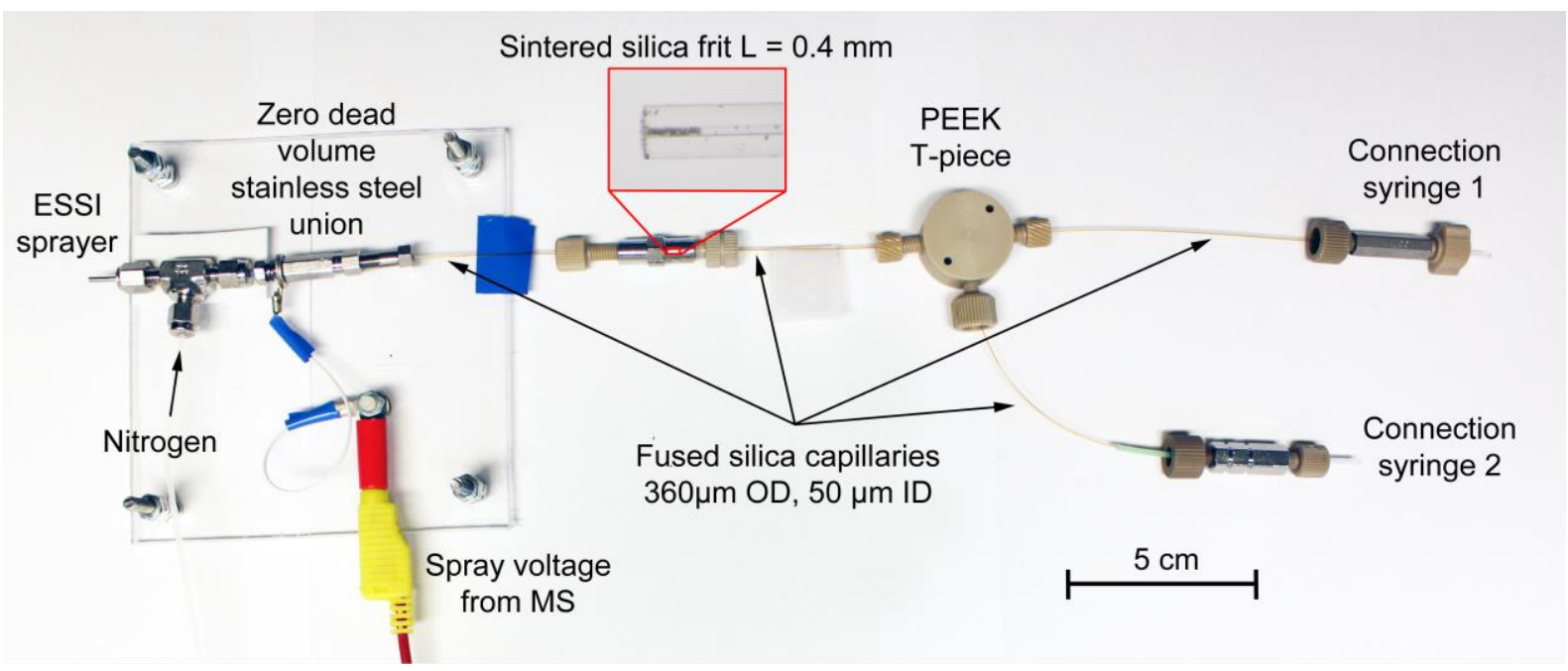

Figure S6. Experimental setup for accelerated pepsin digestion of proteins utilizing ESSI-MS. The ESSI spayer was orthogonally placed in front of the inlet of the mass spectrometer. The sintered frit was produced by tipping the tap of a fused silica capillary ( $360 \mu \mathrm{m} \mathrm{OD,} 50 \mu \mathrm{m}$ ID) into Silica gel 60 for column chromatography ( $15-40 \mu \mathrm{m}$ bead size; Merck, Germany). Sintering was performed by heating the packed section for approx. 10 seconds using a yellow glowing platinum wire. Excess silica beads were removed by flushing the capillary with deionized water. 


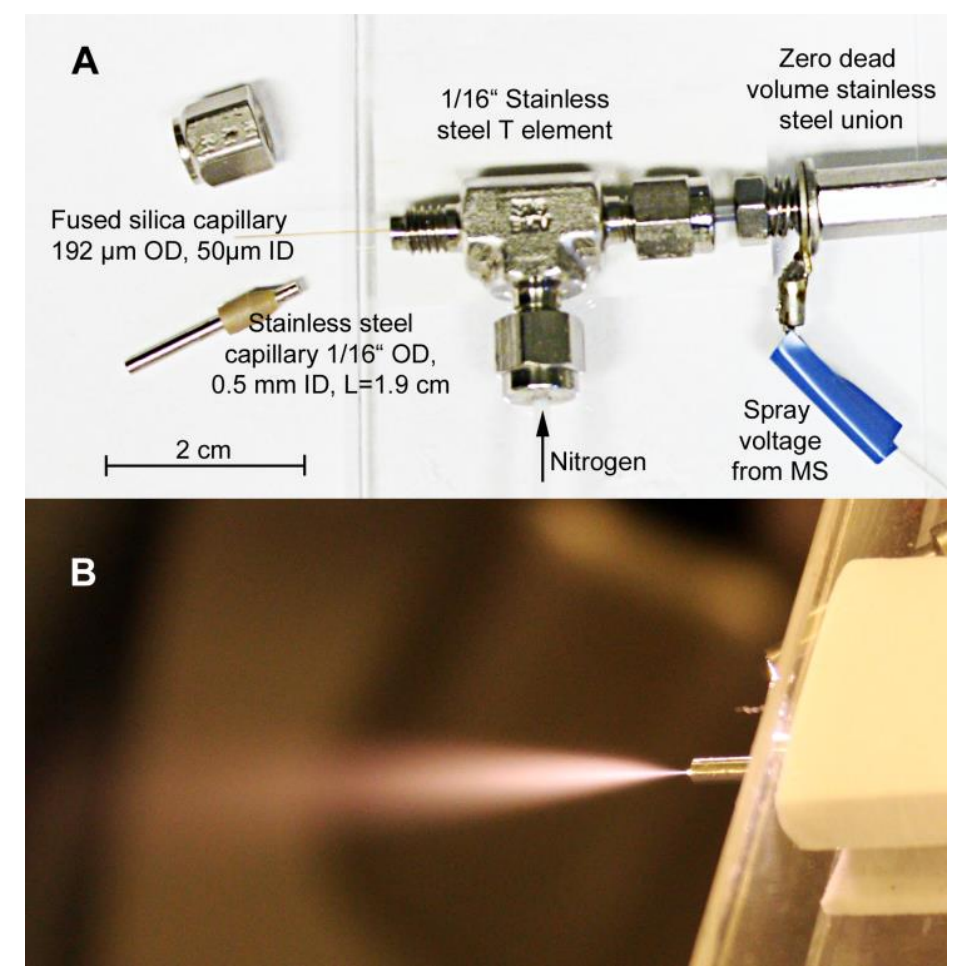

Figure S7. Top: Assembly of the modified ESSI sprayer according to Takáts et al. ${ }^{3}$ Bottom: Photograph of the observed plume at the tip of the ESSI sprayer.

\section{Control experiments}

Bulk digestion of cytochrome c. A vial containing $50 \mu$ l of cytochrome c solution was heated to $95^{\circ} \mathrm{C}$ for $5 \mathrm{~min}$ for denaturation. After cooling to room temperature, $50 \mu \mathrm{l}$ of the pepsin solution was added. The vial was mixed using a vortex mixer. After incubation for 3 hours at $37^{\circ} \mathrm{C}$ using a water bath, the sample was stored at $-20^{\circ} \mathrm{C}$ until ESSI-MS analysis.

Quenching experiment. Experimental setup as depicted in Figure 1B. The two syringes were filled with protein solution Cyt3 as well as protease solutions Pep3. $100 \mu \mathrm{l}$ ammonia $0.5 \%$ quenching buffer $\left(\mathrm{pH}=11.4\right.$ at $\left.22^{\circ} \mathrm{C}\right)$ were placed in an Eppendorf vial on top of a vortex mixer. At a total flow rate of $2.5 \mu \mathrm{l} \cdot \mathrm{min}^{-1}\left(1.25 \mu \mathrm{l} \cdot \mathrm{min}^{-1}\right.$ for each syringe) the stream of liquid was directed into the vial containing the quenching solution. Within $10 \mathrm{~min} 25 \mu \mathrm{l}$ of the eluting reaction mixture were collected in the quenching buffer, the final $\mathrm{pH}$ was checked with $\mathrm{pH}$ test strips and found to be approx. $\mathrm{pH}=8.0$. A $50 \mu \mathrm{l}$ aliquot of the quenched solution was re-acidified with $10 \mu \mathrm{l}$ of $8 \%$ formic acid (to final $\mathrm{pH} \sim 4.5$ ) and submitted to ESSI-MS analysis. Considering the inner volume of a $16 \mathrm{~cm}, 50 \mu \mathrm{m}$ i.d. capillary of approx. 0.3 $\mu \mathrm{l}$, at a flowrate of $2.5 \mu \mathrm{l} \cdot \mathrm{min}^{-1}$, it takes the mixture around 7.5 seconds to pass the capillary from the mixing silica frit into the quenching solution, where the enzyme is irreversibly inactivated. Hence, we assume a reaction time for the digestion in solution of approx. $7.5 \mathrm{sec}$ at a flow rate of $2.5 \mu \mathrm{L} \cdot \mathrm{min}^{-1}$.

\section{References}

(1) Chambers, M. C.; Maclean, B.; Burke, R.; Amodei, D.; Ruderman, D. L.; Neumann, S.; 
Gatto, L.; Fischer, B.; Pratt, B.; Egertson, J.; Hoff, K.; Kessner, D.; Tasman, N.; Shulman, N.; Frewen, B.; Baker, T. A.; Brusniak, M.-Y.; Paulse, C.; Creasy, D.; Flashner, L.; Kani, K.; Moulding, C.; Seymour, S. L.; Nuwaysir, L. M.; Lefebvre, B.; Kuhlmann, F.; Roark, J.; Rainer, P.; Detlev, S.; Hemenway, T.; Huhmer, A.; Langridge, J.; Connolly, B.; Chadick, T.; Holly, K.; Eckels, J.; Deutsch, E. W.; Moritz, R. L.; Katz, J. E.; Agus, D. B.; MacCoss, M.; Tabb, D. L.; Mallick, P. A Cross-Platform Toolkit for Mass Spectrometry and Proteomics. Nat. Biotechnol. 2012, 30 (10), 918-920.

https://doi.org/10.1038/nbt.2377.

(2) Niedermeyer, T. H. J.; Strohalm, M. MMass as a Software Tool for the Annotation of Cyclic Peptide Tandem Mass Spectra. PLoS One 2012, 7 (9), e44913. https://doi.org/10.1371/journal.pone.0044913.

(3) Takáts, Z.; Wiseman, J. M.; Gologan, B.; Cooks, R. G. Electrosonic Spray Ionization. A Gentle Technique for Generating Folded Proteins and Protein Complexes in the Gas Phase and for Studying Ion-Molecule Reactions at Atmospheric Pressure. Anal. Chem. 2004, 76 (14), 4050-4058. https://doi.org/10.1021/ac049848m. 